Margareth Crisóstomo Portela 1 Joyce Mendes de Andrade Schramm 2 Vera Lúcia Edais Pepe 1 Marina Ferreira Noronha 1 Carlos Al berto Marins Pinto 3 Marialva Passos Cianeli 3

\section{Algoritmo para a composição de dados por internação a partir do sistema de informações hospitalares do sistema único de saúde (SIH/SUS) - Composição de dados por internação a partir do SIH/SUS}

\author{
Algorithm for establishing hospital admittance \\ data based on the hospital information system \\ in the Brazilian Unified Health System (SIH/SUS)
}

1 Departamento de Administração e Planejamento em Saúde, Escola Nacional de Saúde Pública, Fundação Oswaldo Cruz.

Av. Leopoldo Bulhões, 1480/717, Manguinhos, Rio de Janeiro, RJ 21041-210, Brasil.

2 Departamento de Epidemi ologia e Métodos Quantitativos, Escola Nacional de Saúde Pública. 3 Secretaria de Saúde do Estado do Rio de Janeiro Rua México 128, 110 andar, Rio de Janeiro, RJ 20031-142, Brasil.

\begin{abstract}
The authorization form for hospital admittance (AlH), an observation unit under the Hospital Information System of the Unified Health System (SIH/SUS), may represent a shortterm hospital admittance or a longer hospital stay. This study presents an algorithm for composing admittance data based on the AlH forms, all owing for a proper assessment of hospital mortality, costs under the Unified Health System (SUS), and Ionger hospital stays, typical of chronic, terminal, and psychiatric patients.

Key words Hospital Information Systems; Algorithms; Medical Informatics

Resumo A autorização de internação hospitalar (AlH), unidade de observação do Sistema de I nformações Hospi talares do SUS (SIH/SUS), pode corresponder a uma internação de caráter agu do ou a um período delimitado de uma internação de longa duração. Este trabal ho apresenta um algoritmo para a composição de dados por internação a partir das AlHs, possibilitando a consi deração adequada da mortalidade hospitalar, custos sob a perspectiva do SUS e tempo de permanência hospitalar em internações de longa permanência, típicas, em especial, entre pacientes crônicos/fora de possi bilidade terapêutica e psi quiátricos.

Palavras-chaves Sistema deInformação Hospitalar; Algorítmos; Informática Médica
\end{abstract}




\section{Introdução}

O Sistema de Informações Hospitalares do Sistema Único de Saúde (SIH/SUS) constitui-se em um banco de dados administrativo, cuja principal finalidade é o pagamento da atenção hospitalar coberta pelo SUS. Apesar de não ter sido desenhado na perspectiva do controle e aval iação da assistência hospitalar, possuindo as limitações inerentes a um banco administrativo neste sentido (Wray et al., 1995), ele tem possibilitado a geração de informações importantes tanto relativas à morbidade, definida em termos das causas principais de internação, quanto à descrição da assistência e do uso de recursos. Também tem sido utilizado em análises da variação da mortalidade e do uso de recursos hospitalares (ex.: tempo de permanência, uso de UTI, procedimentos diagnósticos ou procedimentos de alta complexidade, valores pagos) enquanto função da causa principal de internação, variáveis demográficas, geográficas e relativas à natureza e complexidade do hospital (Martins, 1991; Veras, 1992; Leucovitz \& Pereira, 1993; Gouvêa, 1996; Noronha et al., 1996; Chiyoshi \& Moura, s/d). A recente disponibilização dos dados do SIH/SUS em CDROM e via internet, pelo Ministério da Saúde, vem democratizando o acesso e facilitando a sua incorporação no processo de gestão do sistema de saúde.

\section{Objetivo}

A unidade de observação do SIH/SUS é a autorização de internação hospitalar (AlH), que pode corresponder a uma internação de caráter agudo/curta permanência ou a um período delimitado de uma internação de longa permanência. $\mathrm{O}$ objetivo do trabalho aqui reportado foi propiciar um mecanismo de resgate dos dados de uma internação a partir de dados de diferentes AlHs, possibilitando a construção de um novo banco, cuja unidade de observação fosse a internação hospitalar.

A importância do procedimento proposto fica clara mediante o entendimento da mecânica de emissão de AlHs em internações de Ionga permanência, típicas, em particular, no caso de pacientes crônicos/fora de possibilidade terapêutica (FPT) e psiquiátricos (MS, 1996). Emite-se inicialmente uma AlH do tipo $1 \mathrm{com}$ determinado número, que deve cobrir 45 dias. A partir dos 45 dias, podem ser emitidas até duas AlHs do tipo 5 com o mesmo número da primeira e validade de trinta e um dias cada uma. Se a internação tem continuidade, emite- se uma nova AlH do tipo 1 com outro número, e o processo se repete. No recorte de um ano, uma internação de longa permanência pode então estar relacionada a até onze $\mathrm{AlH}$ s dos tipos 1 e 5 , e até 5 grupos de AlHs de números diferentes. Em conseqüência, análises que não tenham a internação como unidade de observação irão subestimar a mortalidade hospitalar e invalidar a apreciação pertinente de variáveis que traduzem o tempo de permanência hospitalar e custos, na perspectiva do SUS, correspondentes.

Em análises que partem do banco de $\mathrm{AlHs}$, a estratégia de eliminação das AlHs do tipo 5 , para a consideração de internações, repercute em erro desprezível se os diagnósticos ou procedimentos focalizados estão muito raramente associados a uma longa permanência hospitalar. No caso da chance de ocorrência de permanência hospitalar longa ser expressiva, entretanto, a estratégia pode ser desastrosa. Para se dar uma idéia, com base na fita reduzida do SIH/SUS, do total de 4.051 óbitos hospitalares ocorridos entre pacientes crônicos/ FPT, no estado do Rio de Janeiro em 1995, somente 1.810 estavam registrados em AlHs do tipo 1.

A junção de AlHs para a composição dos dados de uma internação de longa permanência é complicada especialmente pela ausência no banco de uma variável que relacione os grupos de Al Hs de diferentes números. O uso do nome do paciente tem sido apontado como alternativa, mas além de não estar publicamente disponível e incorrer em problemas computacionais, envolve considerações éticas relevantes.

$\mathrm{O}$ algoritmo aqui proposto parte de alguns pressupostos lógicos simples, envolvendo, essencialmente, a consideração do número e tipo de AIH, CGC do hospital, data de nascimento, sexo do paciente, diagnóstico principal, procedimento realizado, data de internação, data de saída e motivo de cobrança registrados por AlH. Ele prevê substantiva manipulação de dados arranjados em vetores, sendo as dimensões desses vetores um dos determinantes dos recursos computacionais requeridos. Foi desenvolvido na forma de um programa para uso no pacote estatístico SAS (disponível através dos autores), e experimentado na análise, em computador pessoal, de dados do SIH/SUS relativos ao estado do Rio de Janeiro em 1995. 
Algoritmo utilizado na composição de dados de internações pela junção de AlHs

O processo de construção do banco de dados de internações envolve três etapas: (1) a agregação de AlHs de mesmo número; (2) a recuperação de "datas de nascimento" e "sexo" não especificados, comuns em AlHS do tipo 5; e (3) a junção de grupos de AlHs de números diferentes.

Na primeira etapa são agregadas todas as AlHs de mesmo número em uma única observação, compondo-se novos val ores para as variáveis que discriminam, por exemplo, o total de dias em UTI ou os custos relacionados (vaIor total, valor de serviços auxiliares de diagnóstico e terapêutica, etc.), a partir da soma dos seus respectivos val ores nas Al Hs agregadas. Nesta etapa, para cada observação reunindo um grupo de AlHs de mesmo número, conserva-se a menor data de internação e a maior data de saída registrada.

Na segunda etapa, a recuperação de "datas de nascimento" e "sexo", comumente não especificados em Al Hs do tipo5, é feita a partir de AlHs do tipo 1, de mesmo número, relativas a um período anterior ao período focalizado. No sentido de garantir que dados relacionados correspondam a um único indivíduo em uma única internação, indica-se o uso simultâneo do CGC do hospital e do número da AlH como variáveis-chave na junção dos dados.

A terceira e última etapa do processo de construção do banco de dados de internações está calcada na pressuposi ção de que AlHs resultantes das etapas anteriores, referentes a um mesmo hospital, a uma mesma data de nascimento, a um mesmo gênero (sexo), a um mesmo diagnóstico principal, à realização de um mesmo procedimento e a uma mesma especialidade, compõem uma única internação se, após agrupadas com base nessas variáveis, verifica-se simultaneamente que: (a) a data de internação em uma AlH é a mesma data ou data seguinte àquela de saída da $\mathrm{AlH}$ imediatamente anterior; (b) o motivo de cobrança de AlHS (de números diferentes) seqüenciadas em um agrupamento é dado pelos códigos 13 (alta em estado inalterado), 16 (alta administrativa), 21 (permanência maior que 30 dias por características próprias da doença), 22 (permanência maior que 30 dias por intercorrência), 23 (permanência maior que 30 dias por motivo social), 24 (permanência maior que 30 dias por doença crônica) ou 25 (permanência maior que 30 dias por impossibilidade de vivência sócio-familiar), podendo somente a úl- tima AlH na seqüência passível de compor uma internação estar vinculada a um motivo de cobrança diferente dos mencionados (alta por outras razões, transferência para um outro serviço/clínica ou óbito); e (c) os números de Al Hs consecutivas obedecem à ordem crescente.

\section{Limitações na junção de AlHs}

Fica claro que uma limitação relevante do processo de composição de internações, a partir de AlHs de números diferentes, é a sua incapacidade de dar conta de AlHs que se mantêm com "data de nascimento" e "sexo" desconhecidos. Com exceção de casos muito raros, fica impossível agregar AlHs que sequer apresentam a garantia de poderem estar vinculadas a um mesmo indivíduo.

Uma outra limitação diz respeito à impossibilidade de garantir, em termos absolutos, que os critérios de agregação de Al Hs na última etapa não repercutem em erros. Eles se constituem em pressuposições na medida em que é altamente provável que realmente agrupem AlHs em internações de forma correta; entretanto, não se pode esquecer que eventos altamente improváveis não são necessariamente impossíveis. Em situações que envolvem os procedimentos de politraumatismo e cirurgia múltipla, onde novas cirurgias realizadas em intervalo superior a 24 horas dão origem à emissão de novas AlHs do tipo 1 , a junção de tais AlHs fica impossibilitada se os procedimentos ou diagnósticos principais registrados não são os mesmos.

Vale, finalmente, comentar o uso dos códigos 13 e 16 da variável "motivo de cobrança" como indicadores da continuidade da internação, visto que, rigorosamente, eles deveriam corresponder a uma alta do paciente, com finalização da hospitalização. A nossa opção é justificada pela observação freqüente de tais códigos em Al Hs de crônicos/ pacientes fora de possibilidade terapêutica e de pacientes psiquiátricos que visivelmente devem compor uma única internação. Acredita-se que tal fato é facilmente atribuível a uma interpretação dúbia do que é uma alta em estado inalterado (saída do hospital em estado inalterado ou finalização de uma AlH específica com o paciente em estado inalterado?) ou uma alta administrativa (saída do hospital por motivo administrativo ou finalização burocrática de uma fase - AlH - da internação?). 
Resultados preliminares

e considerações finais

Em análises comparando os números de Al Hs e de internações para os hospitais do município do Rio de Janeiro observou-se redução drástica do número de AlHs para o número de internações nos hospitais cujas principais especialidades são Psiquiatria e crônico/FPT. Naquel es hospitais onde predomina $o$ atendimento nas especialidades de clínica, cirurgia, obstetrícia e pediatria quase não se observa- ram mudanças, sendo o número de AlHs praticamente igual ao número de internações. No que concerne à especialidade crônico/FPT, especificamente, o número de Al Hs 1 e 5 faturadas no Rio de Janeiro, em 1995, correspondeu a 107.304 casos ( $10 \%$ do total de Al Hs), reduzidos a 38.964 na primeira etapa da junção de AlHs e, finalmente, a 27.712 internações (3\% do total de internações).

\section{Referências}

CHIYOSCHI, F. \& MOURA, A., s/d. O Sistema de Assistência Médico-hospitalar da Previdência Social (SAMHPS) - Sistema AlH. (mimeo.)

GOUVA, C. S. D., 1996. Tendências Recentes da Assistência Hospitalar no Estado do Rio de Janeiro. Dissertação de Mestrado, Rio de Janeiro: Escola Nacional de Saúde Pública, Fundação Oswaldo Cruz.

LEU COVITZ, E. \& PEREIRA, T. R. C., 1993. SIH/SUS (Sistema AlH): 1983-1991. SérieEstudos em Saúde Coletiva, 57:1-83.

MS (Ministério da Saúde), 1996. Manual da AlH. In: CD-ROM Movimento de Autorização de Internação Hospitalar - Dezembro/96. Diretório DOCS, Arquivo MANAIH.WRI. Brasília: Ministério da Saúde.

NORONHA, M. F.; MACHADO, C. V. \& LIMA, L. D., 1996. Proposta de indicadores e padrões para a avaliação de qualidade da atenção hospitalar - o caso da asma brônquica. Cadernos de Saúde Pública, 12(supl. 2):43-58.

VERAS, C. M. T., 1992. Equity in the Use of Private Hospitals Contracted by a Compulsory Insurance Scheme in the City of Rio de Janeiro, Brazil, in 1986. Tese de Doutorado, London: Department of Social Sciences and Public Administration, The London School of Economics and Political Science.

WRAY, N. P.; ASHTON, C. M.; KUYKENDALL, D. H. \& HOLLINGSWORTH, J. C., 1995. Using administrative databases to evaluate the quality of medical care: a conceptual framework. Social Science and Medicine, 40:1707-1715. 